\title{
sensors
}

ISSN 1424-8220

(C) 2002 by MDPI

Invited Paper

http://www.mdpi.net/sensors

\section{Mass Transfer in Amperometric Biosensors Based on Nanocomposite Thin Films of Redox Polymers and Oxidoreductases}

\author{
Michael V. Pishko, ${ }^{1,}$ * Alexander Revzin, ${ }^{2}$ Aleksandr L. Simonian ${ }^{2}$ \\ ${ }^{1}$ Department of Chemical Engineering, The Pennsylvania State University, 104 Fenske Laboratory, \\ University Park, PA 16802-4400 \\ ${ }^{2}$ Texas A\&M University, Department of Chemical Engineering, College Station, TX 77843-3122 \\ * Author to whom correspondence should be addressed. Phone: 814-863-4810, Fax: 814-865-7846 \\ E-mail: mpishko@engr.psu.edu
}

Received: 13 February 2002 / Accepted: 28 February 2002 / Published: 12 March 2002

\begin{abstract}
Mass transfer in nanocomposite hydrogel thin films consisting of alternating layers of an organometallic redox polymer (RP) and oxidoreductase enzymes was investigated. Multilayer nanostructures were fabricated on gold surfaces by the deposition of an anionic self-assembled monolayer of 11-mercaptoundecanoic acid, followed by the electrostatic binding of a cationic redox polymer, poly[vinylpyridine Os(bis-bipyridine $)_{2} \mathrm{Cl}$ co-allylamine], and an anionic oxidoreductase. Surface plasmon resonance spectroscopy, Fourier transform infrared external reflection spectroscopy (FTIR-ERS), ellipsometry and electrochemistry were employed to characterize the assembly of these nanocomposite films. Simultaneous SPR/electrochemistry enabled real time observation of the assembly of sensing components, changes in film structure with electrode potential, and the immediate, in situ electrochemical verification of substrate-dependent current upon the addition of enzyme to the multilayer structure. SPR and FTIR-ERS studies also showed no desorption of polymer or enzyme from the nanocomposite structure when stored in aqueous environment occurred over the period of three weeks, suggesting that decreasing in substrate sensitivity were due to loss of enzymatic activity rather than loss of film compounds from the nanostructure.
\end{abstract}

Key words: Biosensor, Redox, Nanocomposite, Hydrogel, Enzyme. 


\section{Introduction}

The electrostatic layer-by-layer assembly of nanocomposite films composed of alternating layers of oppositely charged molecules has been a subject of numerous publications.[1-16] A promising application of this assembly approach is in the area of biosensor fabrication. Layer-by-layer assembly represents a potentially reproducible method to control the architecture of the nanometer-scale biosensing films. In addition, electrostatic assembly of sensing layers is well-suited for mediated electrochemical sensing approaches using charged macromolecular electron acceptor/donors and oxidoreductases. First proposed and implemented by Heller and co-workers,[17] this method entails the electrostatic binding between cationic redox polymers and anionic oxidoreductase enzymes which results in the transfer of electrons from the enzyme via the redox polymer to the electrode surface.[4, 6 , $8,13,14]$ Thus, layer-by-layer assembly of oppositely charged redox polymers and oxidoreductase enzymes takes advantage of this method. Biosensors utilizing various electrostatically complexed redox polymer and enzyme couples have been successfully used to detect glucose, lactate and other analytes. While much attention has been directed to the application of the electrostatically assembled biosensors, few studies have sought to characterize the layer-by-layer electrostatic assembly process or mass transfer within these films. Calvo and co-workers used QCM, AFM, and electrochemical analysis to investigate the assembly process, $[4,6]$ while others employed XPS and SPR for the same purpose. $[6,13]$

In this manuscript we described mass transfer and hydrogel swelling in electrostatically assembled nanocomposite thin films containing redox polymers and oxidoreductases. Surface plasmon resonance spectroscopy (SPR), Fourier transform infrared - external reflection spectroscopy (FTIR-ERS), and ellipsometry were used to characterize film structure. Cyclic voltammetry was used to measure analyte dependent electrode currents that are a function of analyte mass flux in the thin films. While emphasis in this study was placed on the characterization of nanocomposite thin films containing GOX, we have also studied nanocomposite structures containing POD and LOX.

\section{Materials and Methods}

\section{Reagents}

Glucose oxidase (GOX, EC 1.1.3.4, Type X-S, 128 units/mg solid from Aspergillus niger) and lactate oxidase (LAX, 35 units/mg solid, from Pediococcus species) were obtained from the SigmaAldrich Chemical Co. (St. Louis, MO). Pyruvate oxidase (PYX, EC 1.2.3.3, MW 260 kDa) was purchased from Toyobo America, Inc. (New York, NY). 11-mercaptoundecanoic acid (MUA) was obtained from the Aldrich Chemical Co. (Milwaukee, WI). All reagents, unless otherwise stated, were used as received. Phosphate buffered saline (PBS: $10 \mathrm{mM}$ phosphate, $138 \mathrm{mM} \mathrm{NaCl}$, and $2.7 \mathrm{mM}$ $\mathrm{KCl})$ was in $18 \mathrm{M} \Omega \cdot \mathrm{cm}$ distilled, deionized water (E-pure, Barnstead). Silver epoxy was obtained from Epoxy Technologies (Billerica, MA). Silicon wafers coated with $\mathrm{Cr}(20 \AA)$ and $\mathrm{Au}(500 \AA)$ were purchased from Lance Goddard Associates (Foster City, CA).

Polycationic redox polymer, poly[vinylpyridine $\mathrm{Os}$ (bis-bipyridine) ${ }_{2} \mathrm{Cl}$-co-allylamine] (PVP-Os-AA) was synthesized according to the procedures described previously.[8] The structure of this polymer is 
shown in Figure 1. In its reduced and oxidized forms, the oxidation state on the organometallic osmium complex is $+2 /+3$. However, the overall charge on the complex is $+1 /+2$ in its reduced and oxidized states, respectively.

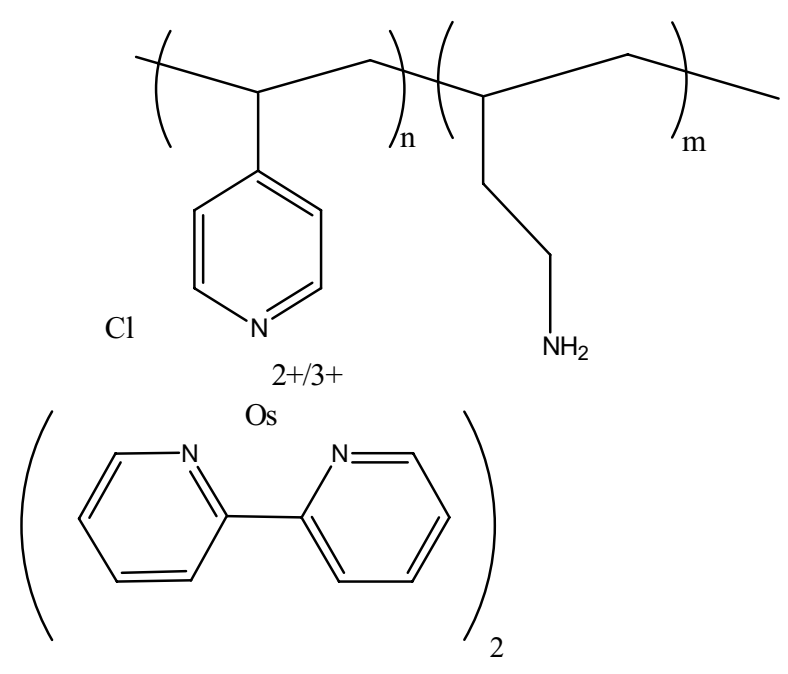

Figure 1. Structure of the polycationic redox polymer used in these studies.

\section{Equipment}

The Spreeta sensor, a miniature (approximately $7 \mathrm{~g}$ ), fully integrated surface plasmon resonance device produced by Texas Instruments, was described in detail elsewhere.[18, 19] Briefly, the sensor possesses a light-emitting diode with a wavelength of $825 \mathrm{~nm}$, a polarizer, a thermistor, and two 128pixel silicon photodiode arrays (EOC 1401). All components are seated on a small printed circuit board and encapsulated with clear, optical epoxy (index of refraction of 1.52), molded to have optically flat surfaces. Because the system was fully encapsulated, no optical alignment is necessary. The molded epoxy is shaped in the form of the Kretschmann geometry prism to facilitate the excitation and detection of surface plasmon resonance. The surface plasmon resonance glass sensing side of the sensor was modified with 10 to $20 \AA$ chromium adhesion layer evaporated onto the surface, followed by the evaporation of $500 \AA$ of gold onto the chromium.

A small flow cell was attached to the sensor to enable delivery of solutions across the sensing surface. In the electrochemical experiments, the gold surface of the SPR substrate also serves as the working electrode. Electrical contact with the gold surface was realized by attaching a wire via conductive silver epoxy; the contacted area was excluded from the flow cell. An additional $\mathrm{Ag} / \mathrm{AgCl}$ reference and Pt counter electrodes were introduced at the top of the flow cell to create a threeelectrode configuration.

Each experiment was performed with a new Au surface. Before starting an experiment, the gold surface of the SPR substrate was thoroughly cleaned using a cotton tip, dipped in $0.1 \%$ Triton and followed by immersion in $0.12 \mathrm{~N} \mathrm{NaOH}$ to remove organic residues on the Au surface. The flow cell was assembled and system was flushed first with ethanol, then with DI water, $0.1 \%$ Triton and $0.12 \mathrm{~N}$ $\mathrm{NaOH}$ solution followed by DI water again. For all experiments the flow cell was equilibrated in running PBS buffer until a stable baseline SPR signal was obtained. All data was acquired using 
refractive index (RI) temperature compensation and each data point was the average of $3-5$ measurements. Experiments were carried out at room temperature $\left(20-21^{\circ} \mathrm{C}\right)$.

Equipment for the electrochemical analysis included a CV-50W Voltammetric Analyzer (Bioanalytical Systems, West Lafayette, IN). The electrochemical apparatus was controlled and data acquired using a Dell Pentium PC. Cyclic voltammetry from 0 to $500 \mathrm{mV}$ (vs. $\mathrm{Ag} / \mathrm{AgCl}$ electrode), at the scan rate of $20 \mathrm{mV} / \mathrm{s}$, was used to measure the substrate-dependent current produced by the redox polymer/ oxidoreductase thin films challenged with varying concentrations of enzyme substrate. Electrodes were tested in a cell of volume approximately $1 \mathrm{~mL}$ containing $0.1 \mathrm{M}$ PBS degassed with $\mathrm{N}_{2}$ (15 min. prior to start of experiment, for duration of experiment) and also containing Pt counter and $\mathrm{Ag} / \mathrm{AgCl}$ reference electrodes. Degassing with $\mathrm{N}_{2}$ agitated the solution created oxygen-free environment.

Ellipsometry was performed using a Gaertner LSE Stokes Ellipsometer (Gaertner Scientific Corp., Chicago, IL). Film thickness and refractive indices were calculated using Gaertner software. All measurements were made using a $\mathrm{He} / \mathrm{Ne}(633.8 \mathrm{~nm})$ laser and a $70.00+/-0.02^{\circ}$ angle of incidence.

\section{Results and Discussions}

We have formed redox polymer and enzyme complexes through an electrostatic layer by layer deposition scheme. This scheme for sensor development minimizes the use of such components as retaining membranes or thick polymer matrices, resulting in an increased surface density of the primary sensing components, namely the enzyme and redox polymer, and thus may potentially improve sensor sensitivity and reduce sensor response time. Minimizing the thickness of the sensing films was achieved by keeping the thickness of the sensing layers well under $100 \AA$ through repeated attachment of single redox polymer/enzyme monolayers.

\section{Thin film assembly and structure}

The scheme used for fabrication of these sensors is based upon electrostatic attraction between a polycationic osmium redox polymer and a polyanionic enzyme, GOX, LAX or PYX. First, the electrode surface was functionalized with MUA where the thiol end group was chemisorbed to gold. The carboxylic acid end group of MUA presented the solution-electrode interface with a net negative charge, since with a pKa of 6.5 the acid group is at least partially deprotonated at a $\mathrm{pH}$ of 7.4.[20] Polymers modified with organometallic osmium complexes are known to be polycationic[8, 14] at neutral $\mathrm{pH}$ and can thus be electrostatically bound to the negatively functionalized surface. Subsequent electrostatic binding of anionic GOX with an isoelectric point of $\sim 3.0$ or LAX with isoelectric point $\sim 4.6[8,14]$ was then possible to the positively charged redox polymer layer. This process was then repeated as desired to deposit multiple layers. A schematic of a final nanocomposite structure is shown in Figure 2.

Figure 3 shows changes in refractive index at the SPR Au surface with time for two binding steps, the initial deposition of RP on the MUA monolayer and the initial deposition of GOX on the RP layer. 


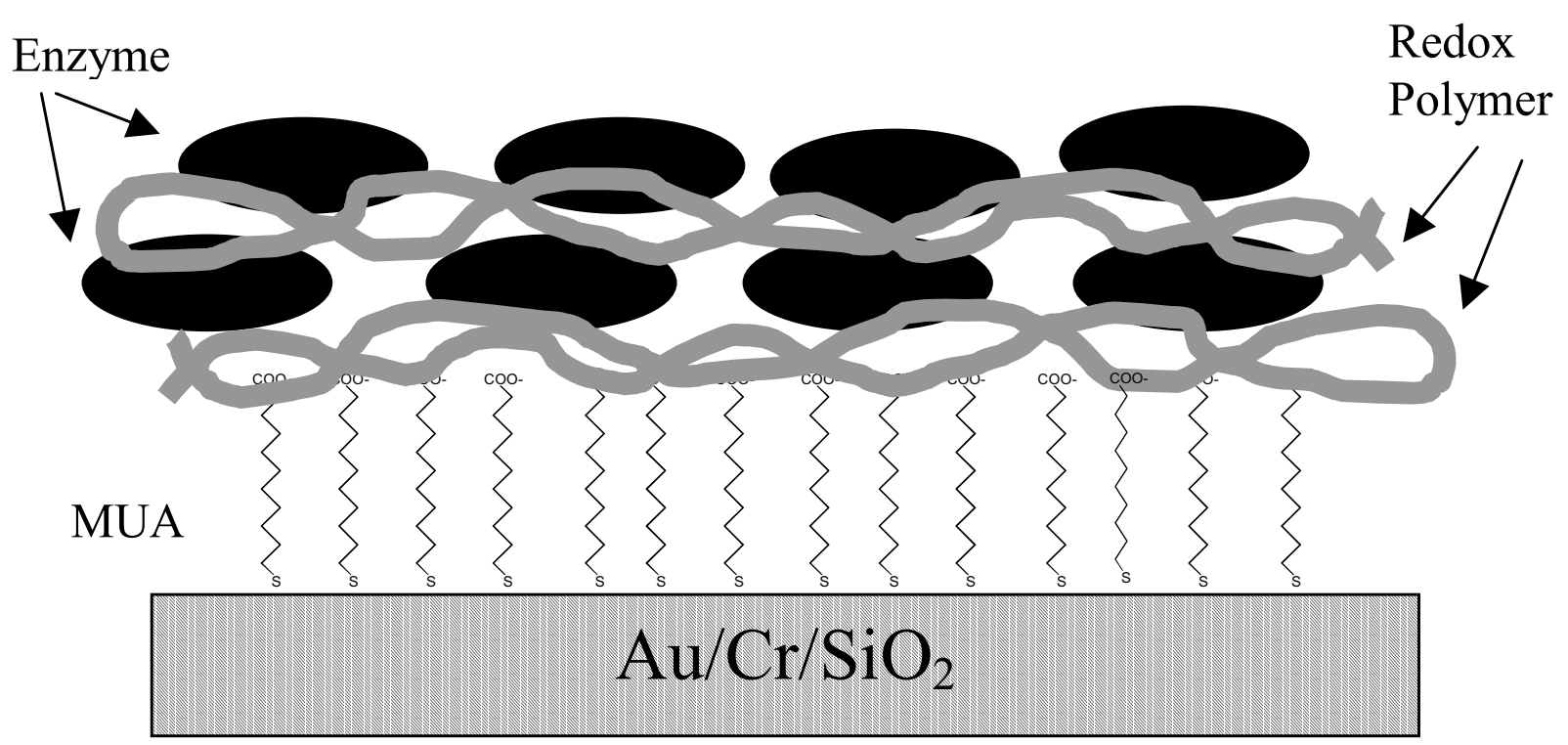

Figure 2. Schematic of the nanocomposite structure created by the layer-by-layer deposition of alternating polyanionic and polycationic films on gold.

The rapid signal increase in the first few seconds after introduction of RP may be attributed to the change in the bulk refractive index of solution.[19, 20] This is followed by a gradual increase that represents the adsorption of material from solution onto the anionic MUA surface. When the surface coverage of RP has reached equilibrium, a plateau in the SPR signal is observed (see Figure 3). At this point, the maximum concentration of polyelectrolyte has adsorbed onto the surface. Once a wash solution is introduced again into the flow-through cell, another sharp change (drop) in SPR response indicates change in the bulk refractive index of solution. This is followed by the desorption of the loosely bound ligand from the charged surface. Thus, a difference between initial and final RI represents the change in RI due to surface bound RP. As can be seen from the SPR signal, this amount of adsorbed RP does not wash from the surface as each RP chain is held in place by multivalent contacts between the polymer chain and the MUA monolayer. Figure 3 also shows a deposition cycle for an oxidoreductase, GOX. Because GOX is a polyanion, it readily adsorbs to the RP layer and again is held in place by multivalent contacts. Continuous SPR monitoring over a 3 week period indicated that these nanocomposite films did not dissociate upon continuous exposure to water. Similar results both in terms of nanocomposite assembly and structural stability were obtained for LAX and PYX, both of which are polyanions at neutral $\mathrm{pH}$. FTIR-ERS confirmed the binding of all enzymes as indicated by the appearance of the amide I and amide II absorption peaks associated with the polypeptide backbone. For all the enzymes, one would expected that the enzyme is highly intercalated with the redox polymer layer, given the highly charged nature of each macromolecule in the multilayer structure.[7, 13, 15, 16, 21, 22]

The SPR signal can be directly related to the surface concentration of proteins with a shift of $\theta_{\text {SPR }}$ by $1^{\circ}$ degree corresponding to the deposition of $10 \mathrm{ng} / \mathrm{mm}^{2}$ of protein.[23] Because the SPR signal can be alternatively recorded in RI units or angle $\theta_{\text {SPR }}$, the surface concentration of enzyme for each deposition step was easily found using the conversion factor of $7 \times 10^{-3} \mathrm{RIU} /$ degree. A surface coverage of $2.4 \mathrm{ng} / \mathrm{mm}^{2}$ in the first GOX assembly step and $1.9 \mathrm{ng} / \mathrm{mm}^{2}$ for the second GOX assembly 
step were determined. These compare favorably with the $3.1 \mathrm{ng} / \mathrm{mm}^{2}$ obtained by Hodak and coworkers in a quartz crystal microbalance study of GOX deposition onto a positively charged gold surface.[6] Surface coverages for LOX and PYX were estimated to be $1.29 \mathrm{ng} / \mathrm{mm}^{2}$ and $10.7 \mathrm{ng} / \mathrm{mm}^{2}$ respectively.

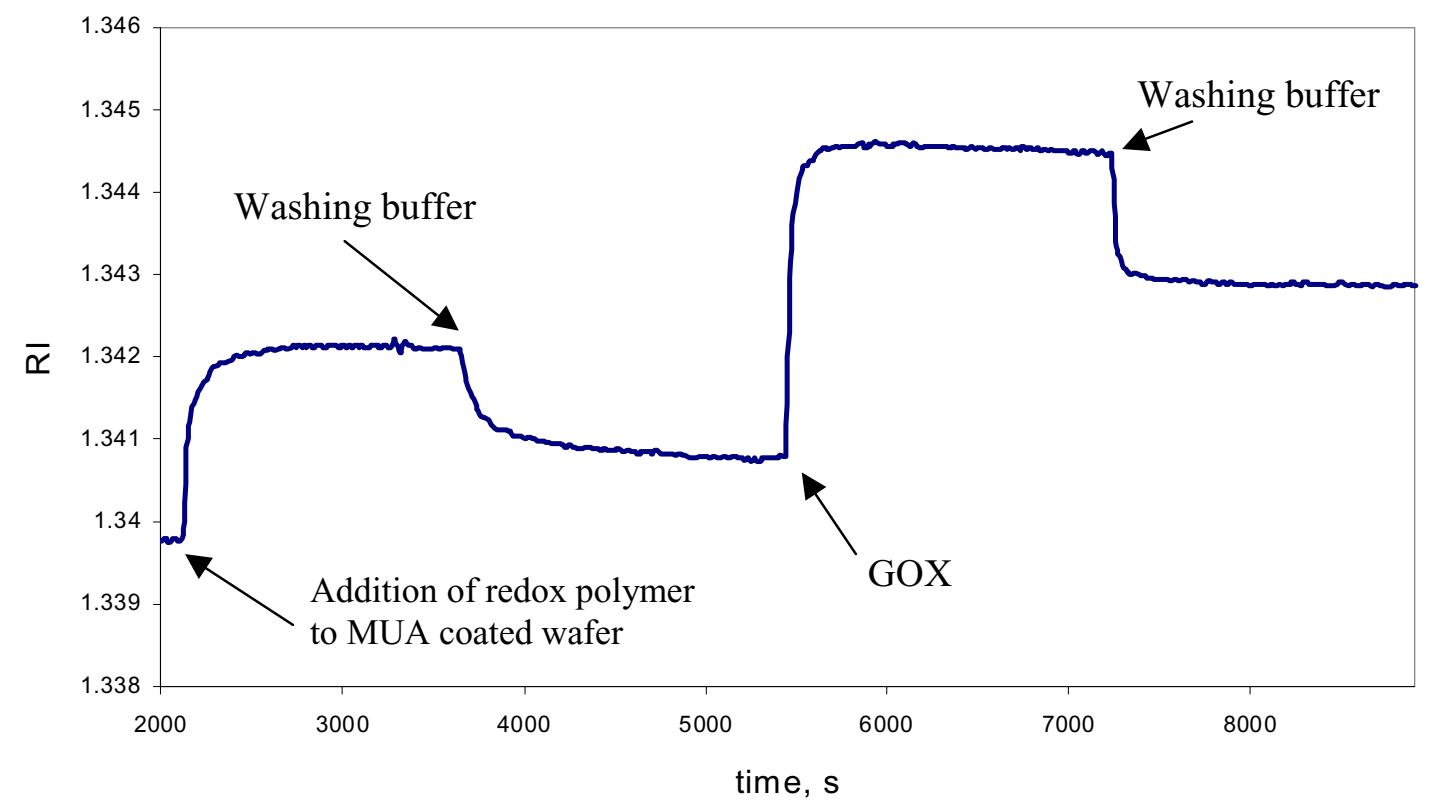

Figure 3. Changes in refractive index with time as measured by SPR during the assembly of a nanocomposite thin film consisting of MUA and one RP/GOX bilayers. The concentrations of RP and GOX were $10 \mathrm{mg} / \mathrm{mL}$ in PBS, flow rate $80 \mu \mathrm{L} / \mathrm{min}$.

Assuming the density of protein to be $1.3 \mathrm{~g} / \mathrm{cm}^{3}$ and a densely packed layer,[12] the thickness of the first deposited enzyme layer was approximately $19 \AA$. While these results do not represent true height of the adsorbed enzyme because surface coverage is not $100 \%$, it may still indicate presence of a monolayer of protein on the positively charged surface. However, given known crystallographic dimensions of GOX,[24] it is highly unlikely that all the adsorbed protein is GOX. This result is not unexpected. All enzyme formulations are contaminated with other proteins and protein fragments of the enzyme of interest. For example, GOX Type XS from Sigma-Aldrich is known to be contaminated with catalase and invertase. In addition, the formulation may likely contain lytic and denatured fragments of GOX and other proteins. Thus the adsorbed protein layer may be a mixture of proteins possessing net negative charge.

The maximum surface coverage (in $\mathrm{ng} / \mathrm{mm}^{2}$ ) for a densely packed enzyme monolayer may be found using the following formula[25]:

$$
P_{\text {ideal }} \sim \frac{10^{21} M W}{\pi a b N_{a}}
$$

where MW is molecular weight (18.6 kDa for GOX, $260 \mathrm{kDa}$ for POD and $80 \mathrm{kDa}$ for LOX), $\mathrm{N}_{\mathrm{a}}$ is Avogadro's number and $\pi a b$ is an ellipsoid surface projection of the globular enzyme, with $a$ and $b$ being the radii of the short and long axis of the ellipse. In case of PYX and LOX, crystallographic data 
does not exist and the ellipsoid surface projection was simplified to $\pi r^{2}$ where $r$ is a hydrodynamic radius. Taking GOX dimensions of $30 \AA$ and $40 \AA$ for $a$ and $b$ respectively,[24] $\mathrm{P}_{\text {ideal }}$ was calculated to be $8.19 \mathrm{ng} / \mathrm{mm}^{2}$. In case of PYX, taking into account $50 \AA$ hydrodynamic radius, $\mathrm{P}_{\text {ideal }}$ was found to be $5.5 \mathrm{ng} / \mathrm{mm}^{2}$. Surface coverage was then found by means of the following relationship:

Surface Coverage $=\left(\frac{P_{\text {expected }}}{P_{\text {ideal }}}\right) \times 100 \%$

Surface coverage estimates for the first two GOX layers were $30 \%$ and $23 \%$ respectively and are reasonable considering that the maximum obtainable coverage for the irreversible adsorption of protein is hypothesized to 55\%.[26, 27] Such low surface coverage is thought to occur because of the steric hindrance and electrostatic repulsion between adsorbing protein molecules.[26] Another explanation may be the fact that lyophilized enzyme powders are not pure (as mentioned earlier) and thus the measurement techniques report an average measurement for all the proteins adsorbed. Thus, one could expect competitive protein adsorption onto the redox polymer surface, which may also account for a lower surface coverage for GOX.

While lack of crystallographic data for LOX does not permit one to approximate surface coverage, one could infer the formation of a sub-monolayer of enzyme from a relatively weak surface coverage. On the other hand, PYX surface coverage is estimated to be $194 \%$. This result may indicate surface aggregation of the less stable PYX tetramer[28, 29] and the formation of multiple enzyme layers in a single PYX deposition step.

As measured by ellipsometry for a dry film, the thickness was $\sim 10 \AA$ for a single GOX layer deposited on a RP layer. This strongly suggests that these nanocomposite thin films are highly swollen by water. One would expect this to be true, given that the nanocomposite films are formed from water soluble polyelectrolytes. Water penetration is also a necessary feature for aqueous electrochemistry in redox polymer thin films. As RP is oxidized, the oxidation state of Os changes from +2 to +3 and thus the charge on the complex changes from +1 to +2 . Electroneutrality must be maintained and thus counter ions must move into the film along with associated water molecules of hydration.

This change in film hydration upon the oxidation and reduction of RP is clearly demonstrated in Figure 4. Here, a nanocomposite film comprised on two RP/GOX bilayers was oxidized and reduced using cyclic voltammetry while simultaneously measuring refractive index using SPR spectroscopy. Upon oxidation at $500 \mathrm{mV}(\mathrm{Ag} / \mathrm{AgCl})$, Os sites on $\mathrm{RP}$ change from +1 to +2 , and counter-ions will move into the film to maintain Electroneutrality. Water associated with these counter-ions will also diffusing in. This flux of water and ions into the nanocomposite film is evident from the decrease in RI as shown in Figure 3 (i.e. the RI in the film moves closer to that of water). When $\mathrm{RP}$ is reduced at $0 \mathrm{mV}$, the charge in the polymer decreases to +1 , water and counter-ions move out of the film, and the film collapses, resulting in an increase in RI (i.e. the RI in the film moves closer to that for a condensed polymer). Because nanocomposite films created from layers of polyelectrolytes have been shown to be nanoporous, the expansion and collapse of the thin film with potential may also represent a change in pore structure and volume at the nanometer level.[16] Fery and colleagues observed changes in nanopore structure based on changes in salt concentration of layerby-layer polyelectrolyte nanocomposites. One may hypothesize that changes in salt concentration as produced by changes in potential may produce a similar effect. 


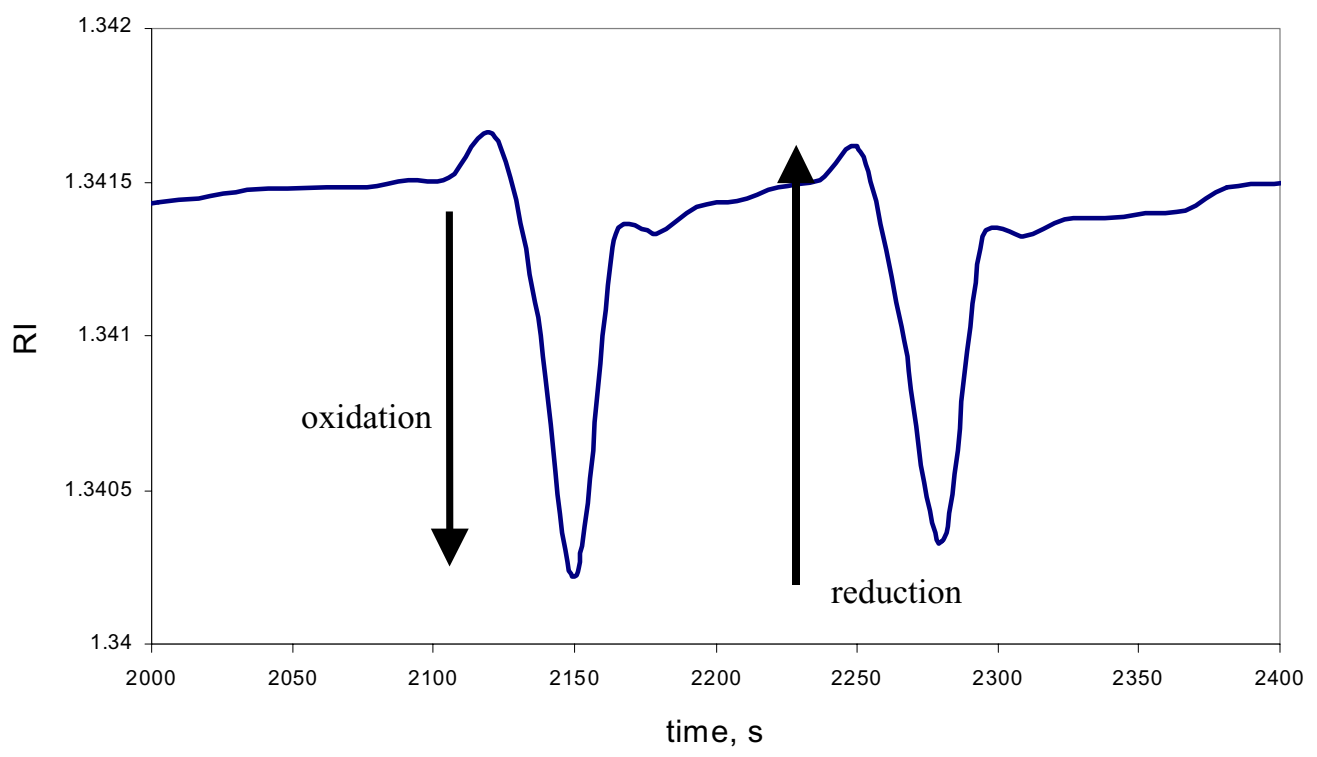

Figure 4. Changes in refractive index with time as measured by SPR during the oxidation and reduction of a nanocomposite thin film consisting of MUA and one RP/GOX bilayers.

\section{Electron transfer and mass transfer at the sensor surface}

The oxidoreductase-catalyzed reaction scheme with Os sites on RP serving as electron relays between the enzyme and electrode surface may be expressed as follows, using glucose oxidase as an example:

$$
\begin{gathered}
\beta \text {-D-glucose }+\mathrm{GOX}(\mathrm{FAD}) \rightarrow \text { Gluconolactone }+\mathrm{GOX}\left(\mathrm{FADH}_{2}\right) \\
\operatorname{GOX}\left(\mathrm{FADH}_{2}\right)+2 \mathrm{M}^{+2} \rightarrow \mathrm{GOX}(\mathrm{FAD})+2 \mathrm{M}^{+1} \\
\mathrm{M}^{+2}+\mathrm{M}^{+1} \rightarrow \mathrm{M}^{+1}+\mathrm{M}^{+2} \\
2 \mathrm{M}^{+1} \rightarrow 2 \mathrm{e}^{-}+2 \mathrm{H}^{+}+2 \mathrm{M}^{+2}
\end{gathered}
$$

where GOX(FAD) and GOX $\left(\mathrm{FADH}_{2}\right)$ represent the oxidized and reduced forms, respectively, of the enzyme's cofactor - flavin adenine dinucleotide (FAD) and M represents an Os redox site of the polymer in its oxidized $(+2)$ and reduced $(+1)$ forms. Electrons are thus transferred from the enzyme to the redox polymer, shuttled between the redox sites in a self-exchange reaction until being transferred to an electrode surface that is poised positive of the formal potential of the polymer. As a result, a current that is proportional to substrate concentration is created. In the absence of mass transfer limitations, the concentration $\left(i=\left(i_{\max }[s]\right) /\left([s]+K_{m}^{a p p}\right)\right)$ dependence of the current is directly related to the Michaelis-Menton kinetics of catalysis for the enzyme, where [s] is the concentration of substrate (e.g. glucose), $\mathrm{i}_{\max }$ is the maximum current possible at high substrate concentrations (analogous to the limiting enzyme turnover rate), and $K_{m}^{a p p}$ is the apparent Michaelis-Menton constant for the enzyme.

Figure 5 shows the concentration dependence of the current density for a nanocomposite thin film modified electrode comprised of two RP/GOX bilayers. The current density was roughly linear in the concentration range from 0 to $20 \mathrm{mM}$ glucose, a result that was not anticipated given that $\mathrm{K}_{\mathrm{m}}$ for $\mathrm{GOX}$ 
is $6 \mathrm{mM}$ [30] and that the film was only several nanometers thickness, making mass transfer limitations unlikely. Nanocomposite films containing LAX and PYX show similar behavior. Figure 5 also shows the anticipated concentration dependence of the current in the absence of mass transfer limitations. As can be seen, mass transfer limitations do exist in these nanocomposite films, a hypothesis supported by several studies. Heller and colleagues constructed micrometer scale polyelectrolyte thin films that limited glucose mass transfer in implanted sensors.[31] Others have observed that polyelectrolyte nanocomposite thin films are highly compact, have a nanoporous structure and can limit the diffusion of small molecules such as ferricyanide.[15, 16, 22] Given that the nanocomposite films described here do undergo significant structural changes upon oxidation and reduction, these films are likely very compact and may posses nanoporosity as seen in other polyelectrolyte thin films.

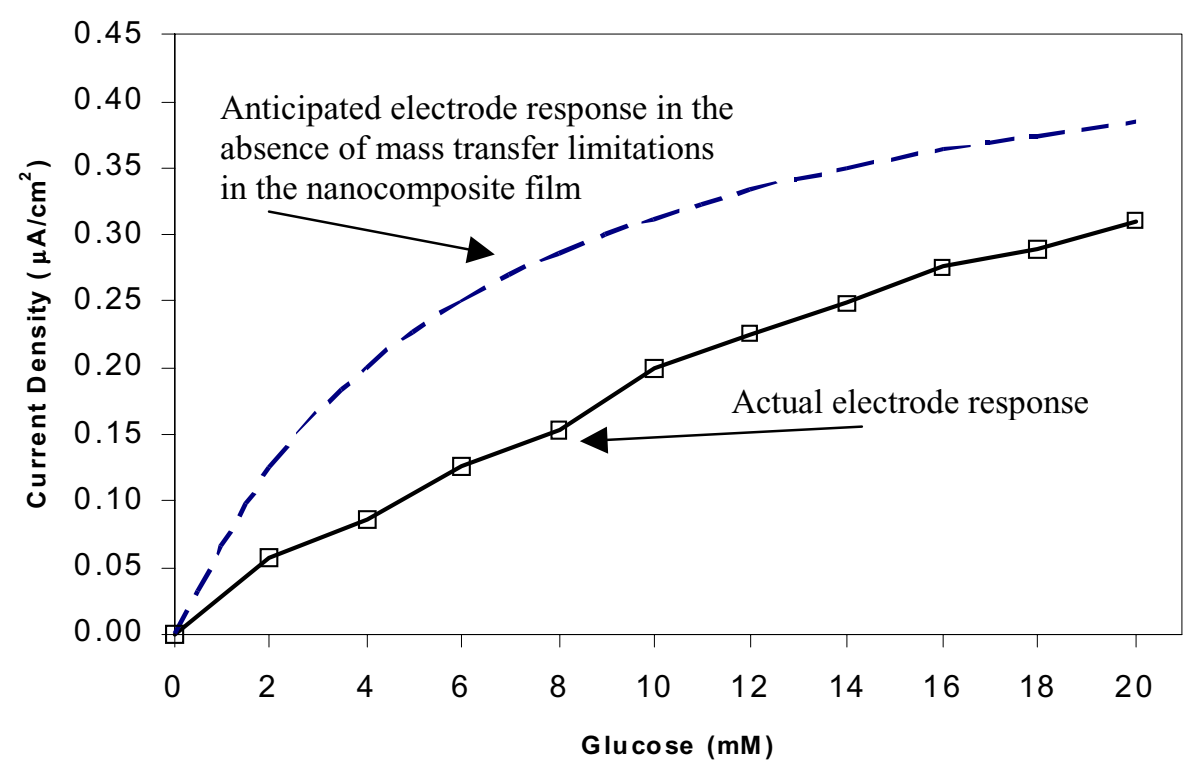

Figure 5. Concentration dependence of the electrode current density for a nanocomposite thin film comprised of two RP/GOX bilayers. The solid line shows the actual response of a nanocomposite thin film while the dash line shows the anticipated response in the absence of mass transfer limitations in the film. Currents were acquired using cyclic voltammetry ( 0 to $500 \mathrm{mV}, 20 \mathrm{mV} / \mathrm{s}$ ), with the current at $500 \mathrm{mV}$ used in the current density calculation.

\section{Conclusions}

In conclusion, we have used SPR and ellipsometry to investigate the layer-by-layer assembly of nanocomposite films on a gold surface. Such investigation is an important step toward better understanding of assembly and properties of enzyme containing nanocomposite thin films. The surface coverages of the deposited GOX, LOX and POD enzymes were estimated by means of SPR spectroscopy. Combined electrochemistry and SPR was used to observe structural changes in the nanocomposite films resulting from the movement of water and ions into and out of the film. The 
concentration dependence of the current for a glucose oxidase-containing nanocomposite film confirmed that the film structure does impose mass transfer limitations, suggesting that it is highly compact and may be nanoporous.

\section{Acknowledgements}

Authors would like to thank Texas Instruments Incorporated and Dr. James Elkind for providing the TISPR-1 sensor and Experimenter's Kit. We would also like to thank Dr. Richard Crooks from the Department of Chemistry at Texas A\&M University and his group members for the use of FTIR-ERS instrument. Financial support was provided by the National Science Foundation (CTS-9875372) and the United States Department of the Army and the National Medical Technology Testbed, Inc. (DAMD17-97-2-7016) for funding. MVP acknowledges the support of the Alfred P. Sloan Foundation through a research fellowship. "This work does not necessarily reflect the position or the policy of the government or NMTB, and that no official endorsement should be inferred."

\section{References}

1. Anicet, N.; Bourdillon, C.; Moiroux, J.; Saveant, J.-M. Electron Transfer in Organized Assemblies of Biomolecules. Step-by-Step Avidin/Biotin Construction and Dynamic Characteristics of a Spatially Ordered Multilayer Enzyme Electrode. J. Phys. Chem. B 1998, 102, 9844-9849.

2. Anzai, J.; Kobayashi, Y.; Nakamura, N.; Nishimura, M.; Hoshi, T. Layer-by-layer construction of multilayer thin films composed of avidin and biotin-labeled poly(amine)s. Langmuir 1999, 15, 221-226.

3. Bacha, S.; Montagne, M.; Bergel, A. Modeling Mass Transfer with Enzymatic Reaction in Electrochemical Multilayer Microreactors. AIChE J. 1996, 42, 2967-2976.

4. Calvo, E.; Battaglini, F.; Danilowicz, C.; Wolosiuk, A.; Otero, M. Layer-by-layer electrostatic deposition of biomolecules on surfaces for molecular recognition, redox mediation and signal generation. Faraday Discuss 2000, 116, 47-65.

5. Clark, S. L.; Hammond, P. T. Engineering the Microfabrication of Layer-by-Layer Thin Films. Adv. Mater. 1998, 10, 1515-1519.

6. Hodak, J.; Etchenique, R.; Calvo, E. J.; Singhal, K.; Bartlett, P. N. Layer-by-Layer Self-Assembly of Glucose Oxidase with a Poly(allylamine)ferrocene Redox Mediator. Langmuir 1997, 13, 27082716.

7. Hou, S.; Yang, K.; Fang, H.; Chen, H. Amperometric glucose enzyme electrode by immobilizing glucose oxidase in multilayers on self-assembled monolayers surface. Talanta 1998, 47, 561-567.

8. Sirkar, K.; Revzin, A.; Pishko, M. V. Glucose and lactate biosensors based on redox polymer/oxidoreductase nanocomposite thin films. Anal. Chem. 2000, 72, 2930-2936.

9. Sun, J.; Wu, T.; Sun, Y.; Wang, Z.; Zhang, X.; Shen, J.; Cao, W. Fabrication of a covalently attached multilayer via photolysis of layer-by-layer self-assembled films containing diazo-resins. Chem. Commun. 1998, 1853-1856. 
10. Sun, Y.; Sun, J. Zhang, X.; Sun, C.; Wang, Y.; Shen, J. Chemically modified electrode via layerby-layer deposition of glucose oxidase (GOD) and polycation-bearing Os complex. Thin Solid Films 1998, 327-329, 730-733.

11. Anzai, J. K.; Suzuki, Y.; Takeshita, H.; Chen, Q.; Osa, T.; Hoshi, T.; Du, X. Enzyme sensors prepared by layer-by-layer deposition of enzymes on a platinum electrode through avidin-biotin interaction. Sensors and Actuators 1998, 52, 3-9.

12. Lvov, Y.; Ariga, K.; Ichinose, I.; Kunitake, T. Assembly of multicomponent protein films by means of electrostatic layer-by-layer adsorption. J. Am. Chem. Soc. 1995, 117, 6117-6123.

13. Laurent, D.; Schlenoff, J. Multilayer Assemblies of Redox Polyelectrolytes. Langmuir 1997, 13, 1552-1557.

14. Revzin, A.; Sirkar, K.; Simonian, A.; Pishko, M. Glucose, Lactate, and Pyruvate Biosensor Arrays Based on Redox Polymer/Oxidoreductase Nanocomposite Thin Films Deposited on Photolithographically Patterned Gold Microelectrodes. Sensors and Actuators 2002, 81, 359-368.

15. Dubas, S.; Schlenoff, J. Swelling and smoothing of polyelectrolyte multilayers by salt. Langmuir 2001, 17, 7725-7727.

16. Fery, A.; Scholer, B.; Cassagneau, T.; Caruso, F. Nanoporous thin films formed by salt-induced structural changes in multilayers of poly(acrylic acid) and poly(allylamine). Langmuir 2001, 17, 3779-3783.

17. Pishko, M. V.; Katakis, I.; Lindquist, S.-E.; Ye, L.; Gregg, B. A.; Heller, A. Direct Electrical Communication between Graphite Electrodes and Surface Adsorbed Glucose Oxidase/Redox Polymer Complexes. Angew. Chem. Intl. Ed. 1990, 29, 82.

18. Melendez, J.; Carr, R.; Bartholomew, D.; Kukanskis, K. Elkind, J.; Yee, S.; Furlong, C.; Woodbury, R.; Spreeta SPR instrument. Sensors and Actuators B 1996, 35-36, 212-216.

19. Elkind, J.; Stimpson, D.; Strong, A.; Bartholomew, D.; Melendez, J. Spreeta SPR instrument. Sensors and Actuators B 1999, 54, 182-190.

20. Franchina, J.; Lackowski, W.; Dermody, D.; Crooks, R.; Bergbreiter, D.; Sirkar, K.; Pishko, M. Entrapment of a biorecognition molecule in a weak-acid, polyelectrolyte hyperbranched thin film on gold. Anal. Chem. 1999, 71, 3133-3139.

21. Hammond, P. T.; Whitesides, G. M. Formation of Polymer Microstructures by Selective Deposition of Polyion Multilayers Using Patterned Self-Assembled Monolayers as a Template. Macromolecules 1995, 28, 7569-7571.

22. G. B. Sukhorukov, J. Schmitt, and G. Decher, Reversible Swelling of Polyanion/Polycation Multilayer Films in Solutions of Different Ionic Strength. Ber. Bunsenges. Phys. Chem. 6 (1996) 948-953.

23. Stenberg, E.; Persson, B.; Roos, H.; Urbaniczky, C. J. Colloid Interf, Sci. 1991, 143, 513-526.

24. Hecht, H.; Kalisz, H.; Hendle, J.; Schmid, R.; Schomburg, D. Crystal structure of glucose oxidase from Aspergillus niger refined at 2.3 A resolution. J. Mol. Biol. 1993, 229, 153-172.

25. Lahiri, J.; Isaacs, L.; Tien, J.; Whitesides, G. A Strategy for the Generation of Surfaces Presenting Ligands for Studies of Binding Based on an Active Ester as a Common Reactive Intermediate: A Surface Plasmon Resonance Study. Anal. Chem. 1999, 71, 777-790.

26. Yuan, Y.; Oberholzer, M.: Lenhoff, A. Colloids and Surfaces 2000, 165, 125-141.

27. Jin, X.; Wang, N.; Tarjus, G.; Talbot, J. J. Phys. Chem. 1993, 97, 4256-4258. 
28. Gajovic, N.; Habermuller, K.; Warsinke, A.; Schuhmann, W.; Scheller, F. A pyruvate oxidase electrode based on electrochemically deposited redox polymer. Electroanalysis 1999, 11, 13771383.

29. Gajovic, N.; Binyamin, G.; Warsinke, A.; Scheller, F.; Heller, A. Operation of a miniature redox hydrogel-based pyruvate sensor in undiluted deoxygenated calf serum. Anal. Chem. 2000, 72, 2963-2968.

30. Frederick, K. R.; Tung, J.; Emerick, R. S.; Masiarz, F. R.; Chamberlain, S. H.; Vasavada, A. Rosenberg, S.; Chakraborty, S.; Schopter, L. M.; Massey, V. Glucose Oxidase from Aspergillus Niger. Cloning, Gene Sequence, Secretion from Sacharomyces Cerevisiae and Kinetic Analysis of a Yeast-Derived Enzyme. J. Biol. Chem. 1990, 265, 3793-3802.

31. Chen, T.; Friedman, K.; Lei, I.; Heller, A. In situ assembled mass-transport controlling micromembranes and their application in implanted amperometric glucose sensors. Anal. Chem. 2000, 72, 3757-3763.

Sample Availability: Available from the authors.

(C) 2002 by MDPI (http://www.mdpi.net). Reproduction is permitted for noncommercial purposes. 